\title{
Nutrition Management of Gestational Diabetes Mellitus
}

\section{Kavita Kapura Anil Kapur ${ }^{\mathrm{b}}$ Moshe Hod ${ }^{\mathrm{c}}$}

${ }^{a}$ Consultant Dietician, Bangalore, India; ${ }^{b}$ World Diabetes Foundation, FIGO Pregnancy and NCD Committee, Bagsvaerd, Denmark; ' Clalit Health Services and Mor Women's Health Center, FIGO Pregnancy and NCD Committee, Tel Aviv, Israel

\section{Key Messages}

- Medical nutrition therapy is the bedrock for managing GDM.

- Many different approaches to nutrition therapy work and are equally effective. More than restriction, it is important to focus on quality of carbohydrates and encourage consumption of vegetables, fruits, complex carbohydrates, and high-fibre foods.

- Monitoring gestational weight gain, self-monitoring of blood glucose and foetal growth is important to modify nutrition advice to achieve optimal outcome for the mother and the newborn.

- Key to success is to provide individualized advice supported by practical tools and training for nutrition self-management and healthy eating and regular follow-up with a dietician or other health care professional trained to provide nutrition counselling.

\section{Keywords}

Gestational diabetes · Management · Nutrition

\begin{abstract}
Medical nutrition therapy (MNT) is the bedrock for the management of gestational diabetes mellitus (GDM). Several different types of dietary approaches are used globally, and there is no consensus among the various professional groups
\end{abstract}

as to what constitutes an ideal approach. The conventional approach of limiting carbohydrates at the cost of increasing energy from the fat source may not be most optimal. Instead, allowing higher levels of complex, low-to-medium glycaemic index carbohydrates and adequate fibre through higher consumption of vegetables and fruits seems more beneficial. No particular diet or dietary protocol is superior to another as shown in several comparative studies. However, in each of these studies, one thing was common - the intervention arm included more intensive diet counselling and more frequent visits to the dieticians. For MNT to work, it is imperative that diet advice and nutrition counselling is provided by a dietician, which is easy to understand and use and includes healthy food options, cooking methods, and practical guidance that empower and motivate to make changes towards a healthy eating pattern. Various simple tools to achieve these objectives are available, and in the absence of qualified dieticians, they can be used to train other health care professionals to provide nutrition counselling to women with GDM. Given the impact of GDM on the future health of the mother and offspring, dietary and lifestyle behaviour changes during pregnancy in women with GDM are not only relevant for immediate pregnancy outcomes, but continued adherence is also important for future health.

(c) 2021 S. Karger AG, Basel karger@karger.com

www.karger.com/anm

() 2021 S. Karger AG, Basel

\section{Karger ${ }^{\prime \prime}=$}




\section{Introduction}

The rising prevalence of gestational diabetes mellitus (GDM) globally and the recognition that medical nutrition therapy (MNT) is the bedrock for its management have led to the search for a pragmatic, feasible, and widely adaptable approach to nutrition therapy to help control maternal glycaemia effectively while also promoting normal foetal growth. The conventional focus so far has been to rigidly limit all types of carbohydrates; though it may help control glucose, it also fosters maternal anxiety and is an important barrier to adherence [1]. Carbohydrates in the form of rice, wheat, pulses, potato, sugar, etc., account for a substantial portion of traditional diets across the world, and limiting their consumption is challenging.

In general, nutrition requirements of women with GDM are similar to non-GDM pregnancies but require a special focus on dietary modification to ensure healthy and mindful eating to achieve and maintain maternal euglycaemia, prevent wide glycaemic excursions, and ensure appropriate gestational weight gain (GWG) and foetal growth. MNT and lifestyle changes are the key elements in the management of GDM. To ensure success of the MNT programme, besides making appropriate adjustments in diet and lifestyle, women with GDM also need to learn about self-monitoring of blood glucose (SMBG) and require education, counselling, emotional support, and regular follow-up [2].

Despite several recent studies, the ideal diet (energy content, carbohydrate restriction, and quality and quantity of macronutrients) for women with GDM remains unclear [3, 4]. While Evert et al. [5] suggest individualizing dietary advice as per the American Diabetes Association guidelines applicable to all persons with diabetes (not restricted to pregnancy) would suffice, the Academy of Nutrition and Dietetics latest clinical guideline states that one type of nutrition plan would not be appropriate for all women with GDM [6], and various national and sub-national strategies based on local culture and eating habits may be needed.

\section{GWG and Energy Intake}

The combination of high pre-pregnancy body mass index (BMI) and excessive weight gain during pregnancy increases the risk of GDM, pre-eclampsia, large for gestational age babies, and complications for both the mother and the newborn at delivery. Overweight or obese pregnant women are also more likely to exceed weight gain recommendations. Furthermore, post-partum weight retention is influenced by the amount of weight gained during pregnancy. Excessive GWG, irrespective of pre-pregnancy BMI, is a significant risk factor for higher fat mass deposit during pregnancy and higher postpartum fat retention [7] which adds to the already high risk of future type 2 diabetes and cardiovascular disease in these women. Overweight and obesity among women with GDM complicate dietary management.

\section{Excessive GWG, irrespective of pre-pregnancy $B M I$, is a significant risk factor for higher fat mass deposit during pregnancy and higher post-partum fat retention}

Globally, the most widely used guideline for GWG is the Institute of Medicine (IOM) guideline [8] which recommends appropriate amount of weight gain per trimester depending on the pre-pregnancy BMI. The IOM guideline does not provide any specific recommendation for women with GDM. The FIGO guideline [9] states that for normal-weight and underweight women, the IOM guidelines apply. Also, usual amount of weight gain and no restriction in calories are recommended to ensure normal infant birth weight. For overweight and obese women, there is no consensus regarding calorie intake and weight gain during GDM pregnancy [9]. There is some evidence to support no weight gain or weight loss in obese women with GDM [10].

Surprisingly, there are very few country-specific guidelines on GWG and most follow the IOM guidelines [11]. Health Canada [12] in their pre-natal guidelines for health professionals has developed a GWG graph using the IOM guidelines to monitor and motivate women to stay within the optimal weight gain range.

There is limited research on caloric requirements and optimum weight gain for women with GDM, and a systemic review of the guidelines from various professional organizations shows varied recommendations. Some recommend between 1,500 and $2,000 \mathrm{kcal} /$ day and others a 30\% calorie restriction for overweight or obese GDM women, while yet another recommends reducing calorie intake by $300 \mathrm{kcal} /$ day [11]. Even though the recommendations of various organizations vary, there is an emerging consensus supporting calorie restriction for overweight and obese women with GDM to avoid excessive GWG [13]. When possible, sequential foetal growth mea- 
surements can provide a more useful benchmark to determine permissible energy intake (EI) in overweight and obese women with GDM than GWG.

According to a WHO report [14], high-risk women who follow lifestyle change interventions (both diet and exercise) reduce the risk of excessive GWG, thereby reducing risk of perinatal complications. In a study by Vestgaard et al. [15], there was relatively lower mean birth weight in newborns of GDM mothers on extended-duration MNT as compared to nonGDM women or GDM women who had no MNT. Birth weight above $4 \mathrm{~kg}$ was seen in 18\% of MNT-treated GDM women versus 27 and 24\% ( $p=0.012$ ) in non-diabetic and no MNT GDM women, respectively. Early diagnosis of GDM and earlier MNT intervention seem beneficial. A Cochrane report states that a combined diet and exercise programme can be useful in preventing GDM in high-risk women [16].

\section{Carbohydrate Restriction}

Carbohydrate restriction remains the most common approach for MNT in GDM. The focus on carbohydrate restriction seems to vary in recommendations from different organizations. The American College of Obstetricians and Gynaecologists (ACOG) [17] and the Endocrine Society [18] recommend restricting carbohydrates in all GDM women on the MNT programme, while the FIGO advises monitoring the carbohydrate intake and the quality of carbohydrates consumed and distributing them throughout the day to attain and maintain euglycaemia [9]. On the amount of permissible carbohydrates, the guidance varies from 35 to $40 \%$ of total calories in the lower carbohydrate range to $50-60 \%$ in the moderate carbohydrate range. However, there seems consensus on not limiting carbohydrate intake to $<175 \mathrm{~g} /$ day (Table 1).

According to Romon et al. [19], carbohydrate restriction to $<39 \%$ may result in higher birth weight. A lower carbohydrate and higher fat and protein intake may increase the risk of GDM in at-risk women [20]. While restricting carbohydrates helps control hyperglycaemia, substituting fat for carbohydrate, especially in obese women with pre-pregnancy insulin resistance (IR), could increase lipolysis and circulating free fatty acids (FFA) available for transplacental transfer leading to excess foetal fat accumulation, as well as worsening maternal IR $[1,21]$ which in turn may worsen hyperglycaemia in the mother [22].

To understand the effect of low carbohydrate on maternal $\mathrm{IR}$, adipose tissue lipolysis, and infant adiposity, a randomized pilot study was undertaken by Hernandez et al. [23]. At 31 weeks, 12 diet-controlled overweight/obese women with GDM were randomized to an isocaloric low-carbohydrate diet (40\% carbohydrate/45\% fat/15\% protein; $n=6$ ) or a higher complex carbohydrate/lower fat (CHOICE) (60\% carbohydrate/25\% fat $/ 15 \%$ protein; $n=6$ ) diet. After 7 weeks on the diet, fasting glucose $(p=0.03)$ and FFAs $(p=0.06)$ decreased in those on the CHOICE diet, whereas fasting glucose increased in those on the low-carbohydrate diet ( $p=0.03)$. The CHOICE diet with higher complex carbohydrates may improve maternal IR and lower infant adiposity [23]. Higher intake of nutrient-dense complex carbohydrates may result in improved metabolic outcomes and reduce excess infant adiposity [24].

In low-carbohydrate diets, the source of fat and protein makes a difference. A pre-pregnancy low-carbohydrate diet with high protein and fat from animal food sources is positively associated with GDM risk, whereas a pre-pregnancy low-carbohydrate dietary pattern with high protein and fat from vegetable food sources is not associated with the risk. Women of reproductive age who follow a low-carbohydrate dietary pattern may consider consuming vegetables rather than animal sources of protein and fat to minimize their risk of GDM [25].

\section{A pre-pregnancy low- carbohydrate diet with high protein and fat from animal food sources is positively associated with GDM risk}

\section{Low-Glycaemic Index Diets}

The type and quality of carbohydrate is an important consideration in nutrition advice for people with diabetes, as not all carbohydrates have the same glycaemic response [26]. The glycaemic index (Gl) of foods is an important factor, as foods with a low Gl reduce post-meal glycaemic excursions and flatten the glucose curve. People with diabetes on high-Gl diets (>70) exhibit higher post-prandial values, and in nonpregnant patients with diabetes, low-Gl diets lead to an additional $0.4 \%$ reduction in haemoglobin A1C [27].

Besides the conventional advice of restricting carbohydrates, studies demonstrate an important role for low-Gl diets in GDM [13]. In fact, in GDM, diets higher in unrefined/complex carbohydrates have been shown to effectively blunt post-prandial glycaemia $[28,29]$, reduce the need for insulin 


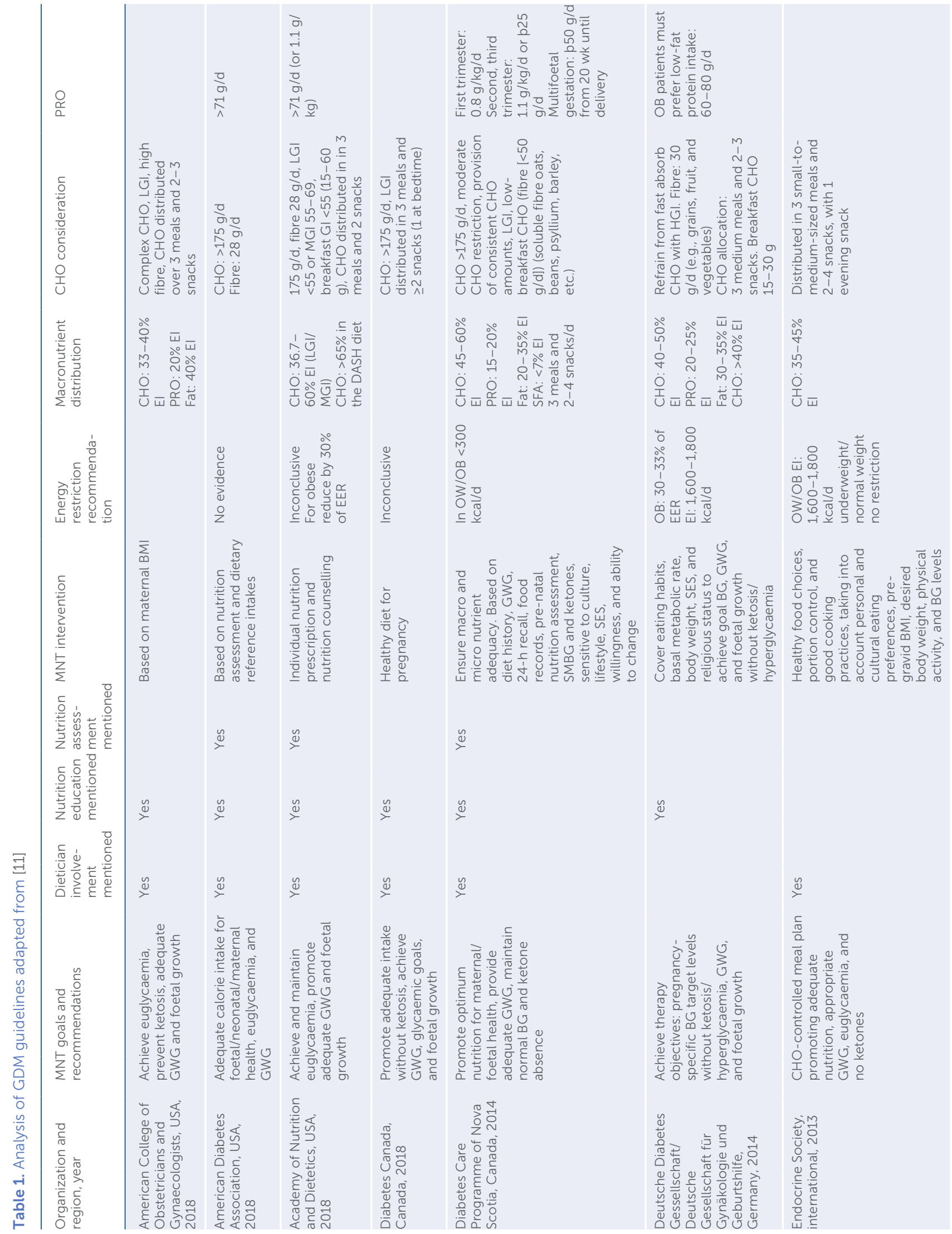









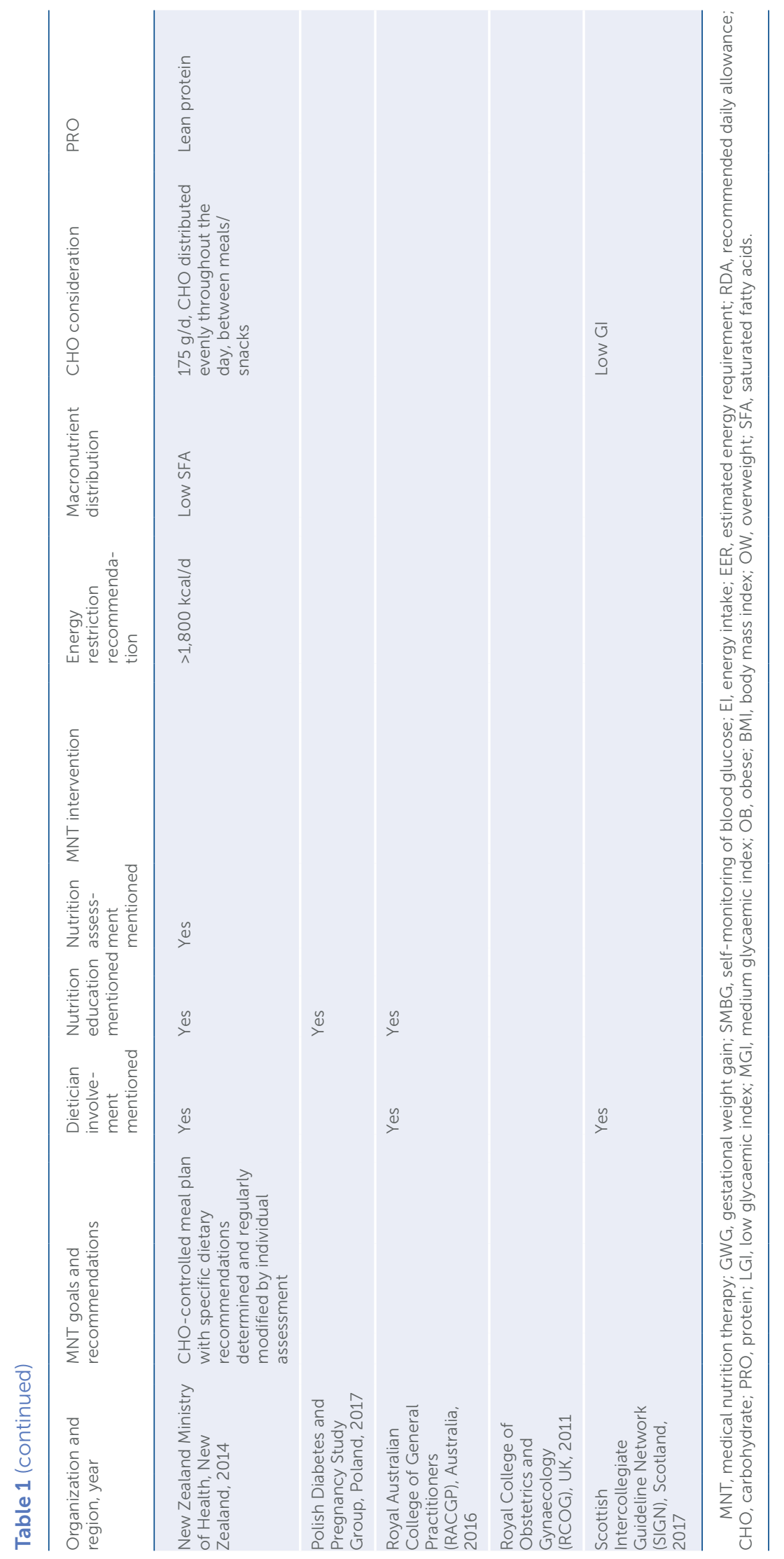


therapy [30], lower fasting LDL cholesterol levels [28, 31] and FFAs [28], and improve insulin sensitivity [32], $\mathrm{HbA}_{1} \mathrm{C}$ [31], and systolic blood pressure [31].

The role of low-Gl diets in GDM has been extensively studied. A meta-analysis of 5 randomized clinical trials with 302 participants studied the effect of low Gl versus control diets and found that low-Gl diets reduced the risk of macrosomia in women with GDM and low-Gl diets with added dietary fibre reduced usage of insulin [33]. The key effect of low-Gl diet was reduction in 2-h post-prandial glucose, fasting plasma glucose, and lipid profile in women with GDM and a substantial decrease in insulin requirement [34].

Another meta-analysis of 11 trials involving 1,985 women evaluated both maternal and newborn outcomes. Low-Gl diet was shown to have a positive effect on maternal outcomes even for those at risk of hyperglycaemia without adverse outcomes on newborns [35].

Three recent meta-analysis and systematic reviews studied various diets and pregnancy outcomes. Viana et al. [36] and Wei et al. [33] concluded that low-Gl diets were associated with a decreased risk of infant macrosomia, whereas a Cochrane review, including 19 trials randomizing 1,398 women, found no clear difference in large for gestational age or other primary neonatal outcomes with the low-Gl diet [37]. It is nonetheless important to note that more than 9 guidelines on nutrition recommendation for GDM from professional organizations recommend a low-to-moderate Gl diet [11].

\section{Dietary Fibre}

Fibre intake, particularly soluble fibre, is beneficial in lowering serum lipid levels and reducing glycaemic excursions. Low-Gl foods often have higher fibre content, but that is not always the case. High-fibre foods in a mixed meal can serve the same purpose as low-Gl diets.

To understand the difference between low-Gl diets and high-fibre diets, 139 women at high risk of GDM (mean [SD] age: 34.7 [0.4] years and pre-pregnancy BMI: $25.2[0.5] \mathrm{kg} / \mathrm{m}^{2}$ ) were randomly assigned to either a low-Gl diet (Gl target 50) or a high-fibre, moderate-Gl diet (target Gl 60) during 1420 weeks of gestation. The average daily amount of fibre intake in each diet group was not stated. Similar pregnancy outcomes (glycosylated haemoglobin, fructosamine or lipids at 36 weeks, or differences in birth weight, Ponderal index birth weight centile, \% fat mass, or incidence of GDM) were seen in both groups [38].

No good quality studies on the benefits of fibre-rich diets in women with GDM are available; it is however recommended that foods rich in fibre should be preferred. Up to 28 -g fibre intake per day is recommended for women. Fibre also helps reduce constipation, a common problem in pregnancy. Apart from the use of low-Gl and high-fibre diets, another commonly used method to reduce high post-prandial levels and wide post-meal glucose excursions and high fasting glucose, recommended by most guidelines, is distributing the total daily allocated carbohydrate portions into 3 small meals and $2-3$ snacks per day [11].

\section{Fat}

MNT in GDM has primarily focussed on control of maternal glycaemia; however, data suggest that maternal lipids, especially triglycerides, may be stronger drivers of foetal growth than glucose $[39,40]$.

\section{Maternal lipids, especially triglycerides, may be stronger drivers of foetal growth than glucose}

Increased consumption of total and saturated fat could worsen IR (Barbour LA, 2007) and increase foetal nutrient exposure, promoting overgrowth patterns. In a randomized study of women with GDM, CHO restriction (40\% of total calories, compared to $60 \%$ complex $\mathrm{CHO}$ ) was accompanied by 20\% higher post-prandial FFAs [41].

It is therefore important that the fat content total and saturated fat of diets of women with GDM need to be moderated. Most GDM guidelines of various professional organizations (Table 1 ) do not specify the amount of recommended fat intake; amongst those who do, there is a wide variation in recommendations. Most fall in the range of $20-35 \%$ of daily El with the ACOG guideline being the outlier recommending up to $40 \%$ of daily El.

\section{Protein}

Adequate protein intake during pregnancy is essential to prevent depletion of maternal stores and prevent muscle breakdown to supply for the foetal needs. Most nutrition guidelines recommend a protein intake between 10 and $20 \%$ of daily El and between 60 and $80 \mathrm{~g}$ of protein intake 
daily. The Indian guidelines recommend a minimum additional $23 \mathrm{~g}$ of protein intake daily during pregnancy over and above the normal recommended daily allowance for adult women. Protein intake restrictions may be required in presence of renal failure.

Several specialized dietary protocols have also been tested in women with GDM. Some of these studies are briefly described below.

\section{DASH Diet}

A randomized controlled trial was conducted to study the effects of the DASH (Dietary Approaches to Stop Hypertension) diet on pregnancy outcomes in women with GDM. Fifty-two participants were randomly assigned to either the control diet or DASH diet for 4 weeks. The control diet contained $45-55 \%$ carbohydrates, $15-20 \%$ protein, and $25-30 \%$ total fat while the DASH diet was rich in fruits, vegetables, whole grains, and low-fat dairy products and contained lower amounts of saturated fats, cholesterol, and refined grains with a total of 2,400 $\mathrm{mg} /$ day sodium. Participants on the DASH diet had better metabolic outcomes than those in the control group. Also, infants born to mothers on the DASH diet had significantly lower weight, head circumference, and Ponderal index compared with those born to mothers on the control diet. Only $46.2 \%$ of women in the DASH diet group needed caesarean section as compared to $80.8 \%(p<0.01)$ in the control group. Similarly, only $23 \%$ participants on the DASH diet needed insulin therapy as compared to 73\% for the control group ( $p<$ 0.0001) [42].

\section{Mediterranean Diet}

The Mediterranean diet was studied as part of the St Carlos GDM Prevention Study - a prospective randomized study wherein both the intervention group and control group were given the same basic Mediterranean diet (MedDiet) recommendations of 2 servings/day of vegetables, 3 servings/day of fruit (avoiding juices), 3 servings/day of skimmed dairy products and wholegrain cereals, $2-3$ servings of legumes/week, moderate to high consumption of fish, and a low consumption of red and processed meat and avoidance of refined grains, processed baked goods, pre-sliced bread, soft drinks and fresh juices, fast foods, and precooked meals. All GDM participants were advised Mediterranean diets plus a recommended daily extra virgin olive oil intake $\geq 40 \mathrm{~mL}$ and a daily handful of nuts. Results showed that the intervention group had reduced incidence of GDM and improved several mater- nal and neonatal outcomes [43]. Mediterranean diet intervention advised early in the pregnancy or to pre-pregnant women has been shown to reduce GDM incidence and maternalfoetal adverse outcomes $[44,45]$.

\section{Non-Nutritive Sweeteners}

Several non-nutritive sweeteners have become available and are widely used by women, but their use during pregnancy has not been well studied, and there is still no clear understanding on their use in pregnancy, with only a couple of international guidelines approving the use of some of them during pregnancy. Aspartame, saccharin, acesulfame, and sucralose are recommended by a few guidelines in moderate amounts. Besides the above, the Academy of Nutrition and Dietetics also accepts usage of advantame, neotame, luo han guo extracts, and steviol glycosides as per the FDA ADI limits. Cyclamates are not approved [11].

\section{Interventions to Prevent GDM - Probiotics and Myoinositol}

Preventing GDM could have several benefits such as reduction in the immediate adverse outcomes during pregnancy, a reduced risk of long-term sequelae, and a decrease in the economic burden to health care systems. Current available evidence about the prevention of GDM showed that the majority of the interventions done during pregnancy have nonsignificant effect in preventing GDM $[46,47]$. Dietary intervention can reduce the risk of developing GDM and the proportion of infants born with macrosomia among pregnant women with obesity; physical activity interventions have not had the same effect. However, conclusive evidence is not yet available to guide practice $[48,49]$. Supplement interventions with probiotics and myoinositol during pregnancy showed a decrease in the rates of GDM compared with a placebo [47, 50]. Intervention showed that probiotics (Lactobacillus rhamnosus and Bifidobacterium lactis Bb12) reduced the incidence of GDM, from 36 to $13 \%$; probiotic consumption may protect against GDM because these microorganisms can modify intestinal microbiota, altering the fermentation of dietary polysaccharides and improving intestinal barrier function [50]. Moreover, myoinositol supplements ( $4 \mathrm{~g}$ ) were found to reduce $50-60 \%$ of the incidence of GDM in high-risk pregnant women (overweight, obese, or first-degree relative of type 2 diabetes mellitus) $[47,51]$. Myoinositol, an isomer of inositol, is one of the intracellular mediators of the insulin signal and correlated with insulin sensitivity in type 2 diabetes. The po- 
Table 2. Signal system [56]

\begin{tabular}{|c|c|c|c|}
\hline Principles & Green & Yellow & ed \\
\hline Refined cereals and sugars & Low & Moderate to high & High \\
\hline Total fat & Low & Moderate & High \\
\hline Glycaemic index & Low & Moderate to high & High \\
\hline Cooking method & $\begin{array}{l}\text { Steaming, boiling, roasting, } \\
\text { grilling, less fat in cooking }\end{array}$ & $\begin{array}{l}\text { Pan fried, sautéed, moderate } \\
\text { amount of fat in cooking }\end{array}$ & $\begin{array}{l}\text { Deep fried, rich in fat and sugar, } \\
\text { rich sauce/cream dressing }\end{array}$ \\
\hline Processing & Rich in fibre, parboiled & Low fibre, refined, milled & $\begin{array}{l}\text { Low fibre, ready to eat, highly } \\
\text { processed }\end{array}$ \\
\hline
\end{tabular}

tential beneficial effect on improving insulin sensitivity suggests that myoinositol may be useful for women in preventing GDM. In conclusion, in women at high risk of developing GDM, the current evidence has showed that dietary advice, probiotics, and myoinositol supplementation might reduce the incidence of GDM.

\section{Interventions to Enhance Healthy Eating and Meal Planning}

Systematic reviews studying 19 trials and comparing the effects of 10 different types of dietary advice for women with GDM found no conclusive evidence to show superiority of one approach or diet programme over others [37]. These included studies comparing a low-to-moderate Gl diet versus a moderate high-Gl diet; an energy-restricted diet versus no energy restriction; a DASH diet versus a control diet; a lowcarbohydrate diet versus a high-carbohydrate diet; a highunsaturated fat diet versus a low-unsaturated fat diet; a lowGl diet versus a high-fibre moderate-Gl diet; diet recommendations and diet-related behavioural advice versus diet recommendations only; a soy protein-enriched diet versus no soy protein; a high-fibre diet versus a standard-fibre diet; and an ethnic-specific diet versus a standard healthy diet.

However, other meta-analyses show that the low-Gl diet, characterized by intake of high-quality, complex carbohydrates, demonstrated lower insulin use and reduced risk of macrosomia. Recent evidence suggests the Mediterranean diet is safe in pregnancy [52]. In developing countries, a oneon-one simple dietary advice for higher consumption of whole grain, dairy products, and dietary fibre was inversely associated with adverse neonatal outcomes in women with GDM [53].

Hrolfsdottir et al. [54] recommend a simple dietary screening questionnaire given early in the first trimester to help identify women with high-risk eating habits associated with GDM and providing individualized dietary feedback and advice. This could help improve eating habits and better manage the pregnancy.

Pregnancy provides an eminent window of opportunity for changing behaviour towards healthy eating and lifestyle and is considered a wonderful teachable moment for women and their families. Change preparedness is high, as emotion is increased because of perceived risk but with the possibility of improved outcome with change. There is also greater motivation, sense of self-efficacy, and willingness to acquire new skills. This is particularly relevant for GDM pregnancy where nutrition and lifestyle change provides the bedrock for managing the condition. Given the impact of GDM on the future health of the mother and offspring, these changes are not only relevant for the immediate pregnancy outcomes, but continued adherence is also important for future health. Despite this, adherence to nutrition advice is often less than satisfactory. An important barrier for non-adherence is the difficult to understand, impractical, and prescriptive advice that is often given, rather than advice that is practical, contextual, and empowers women to make healthy choices. Most of the modifiable barriers to improving adherence to diet are related to nutrition self-management training and counselling skills of care providers [55].

No particular diet or dietary protocol is superior to another as mentioned earlier. However, in each of the studies evaluating different dietary protocols, one thing was common - 


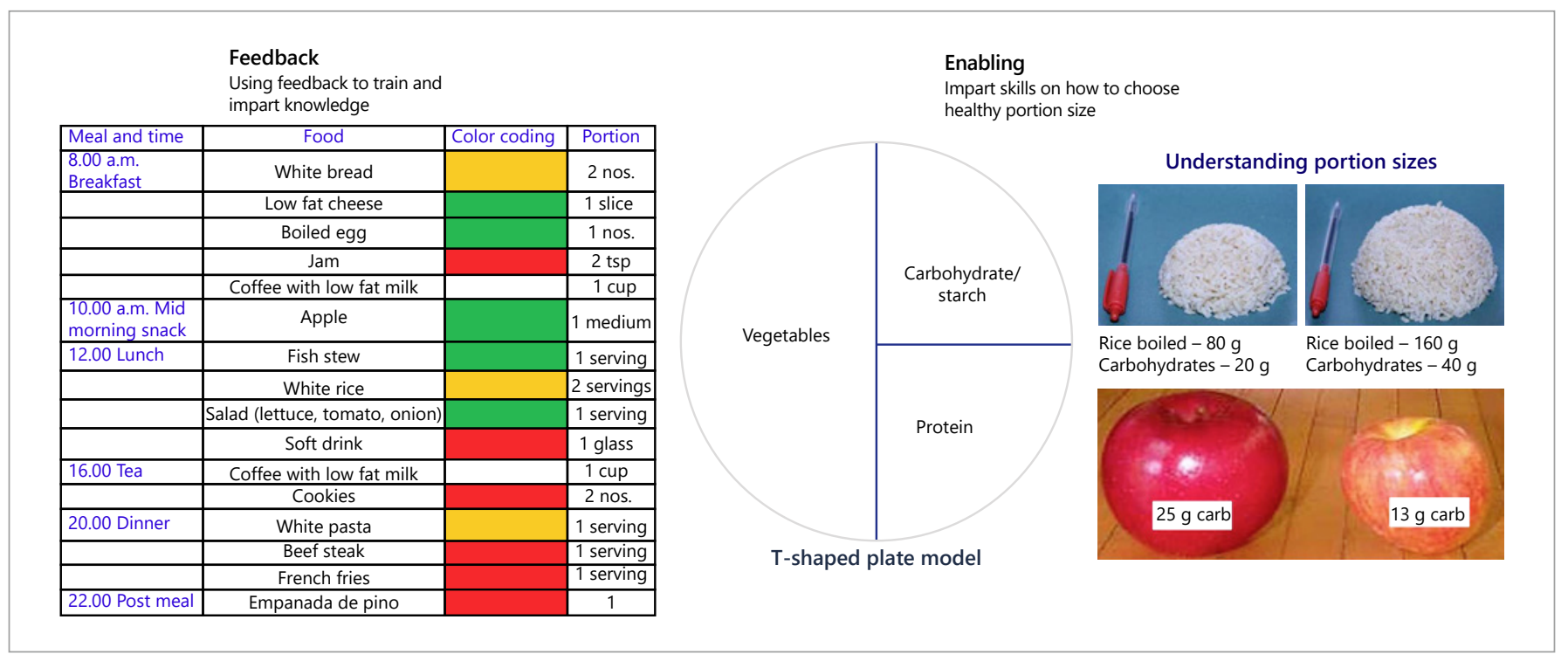

Fig. 1. Tools to support sustained behaviour change.

the intervention arm included more intensive diet counselling and more frequent visits to the dieticians. Advice given by a qualified dietician, more frequent visits to a dietician, advice that includes elements to promote overall health not merely control of blood sugar, nutrition counselling that is easy to understand and use and includes healthy food options, cooking methods, and practical guidance to deal with lifestyle issues are the most important facilitators to improve nutrition advice adherence [55].

Several easy-to-implement tools are available to make nutrition counselling more effective. Some of these are discussed below.

\section{Signal System}

The signal system is an easy-to-use educational tool which highlights the basic principle of healthy food and healthy cooking methods to help patients make informed choices. It follows a simple traffic light concept of red for "stop," yellow for "go slow," and green for "go" and helps people make informed choices on which foods are healthy and which are not. The signal system focuses not only on the number of calories and fat in the food, glycaemic load, and fibre content of food but also on the cooking and processing method [56]. It is similar to the traffic light diet promoted in Australia [57] for healthy eating.

Mapping foods according to red, yellow, and green colour codes helps in educating people on healthy and not so healthy foods and how processing and different cooking methods impact foods making healthy foods unhealthy. It has been used as an educational tool which can be easily adapted to different regional and local foods across the world [56]. The basic principle of the signal system is shown in Table 2.

\section{Portion Size}

The T-shaped plate model especially for the main meals is effective as a basic teaching tool to control portion size and plan meals more effectively (Fig. 1). The healthy plate models are simple and accessible and help enhance the consumption of fruits and vegetables [58]. Visuals of portion sizes and use of household containers (cups and glass) as measures of food quantity are practical and easy teaching tools to help improve adherence to quantity of food consumed.

\section{Food Exchange Tables}

Food exchange tables is a great tool to enhance individualization of dietary advice as it empowers patients to add variety to the prescribed meal plan while at the same time ensuring a balanced intake of all necessary nutrients. A retrospective cohort study showed reduced adverse events in the group receiving MNT using the food exchange tables as compared to the group not receiving MNT [59].

\section{Food Journal}

Maintaining a food journal and SMBG records and analyzing them together help to understand the effect of different foods on glucose levels, to adjust the diet to change portion size of carbohydrates in different meals, and to improve glycaemic control. 


\section{Individualizing Diet Advice}

A simple two-step process to individualize dietary advice is as follows:

- Step 1: Identifying both the good eating habits and not so good eating habits in the current eating pattern of the patient by colour coding the diet history using the signal system/traffic light concept (see Fig. 1). Understanding the portion sizes for each food consumed. Using the colourcoded diet history to discuss and emphasize the good eating habits as well as identify unhealthy patterns. Are the portion sizes appropriate? What are the sources for starch and their quantity (bread, rice, pulses, potato, sweetened beverages, juice, and sugar)? Are source and amount of fat and salt intake in the diet at large? Are there sufficient vegetables and fruit in the diet? The information gathered is used to first praise and motivate the patient on positive aspects while highlighting the need to change unhealthy eating habits.

- Step 2: Using shared decision-making skills, negotiating goals, and keeping the patient's target in mind to encour-

\section{References}

1 Hernandez TL. Carbohydrate content in the GDM diet: two views: view 1: nutrition therapy in gestational diabetes: the case for complex carbohydrates. Diabetes Spectr. 2016;29(2):82-8.

2 Reader DM. Medical nutrition therapy and lifestyle interventions. Diabetes Care. 2007;30(Suppl 2):S188-93.

3 Kim C. Gestational diabetes: risks, management, and treatment options. Int J Womens Health. 2010;2(2):339-51.

4 Han S, Middleton P, Shepherd E, Van Ryswyk E, Crowther CA. Different types of dietary advice for women with gestational diabetes mellitus. Cochrane Database Syst Rev. 2017;2:CD009275.

5 Evert AB, Boucher JL, Cypress M, Dunbar SA, Franz MJ, MayerDavis EJ, et al. Nutrition therapy recommendations for the management of adults with diabetes. Diabetes Care. 2013;36(11): 3821-42

6 Duarte-Gardea MO, Gonzales-Pacheco DM, Reader DM, Thomas AM, Wang SR, Gregory RP, et al. Academy of nutrition and dietetics gestational diabetes evidence-based nutrition practice guideline. J Acad Nutr Diet. 2018;118(9):1719-42.

7 Subhan FB, Shulman L, Yuan Y, McCargar LJ, Kong L, Bell RC: APrON Study Team and ENRICH. Association of pre-pregnancy $\mathrm{BMI}$ and gestational weight gain with fat mass distribution and accretion during pregnancy and early postpartum: a prospective study of Albertan women. BMJ Open. 2019;9(7):e026908.

8 Institute of Medicine. Implementing guidelines on weight gain and pregnancy. Bethesda, MD: Institute of Medicine and National Research Council; 2013.

9 Hod M, Kapur A, Sacks DA, Hadar E, Agarwal M, Carlo Di, et al. The International Federation of Gynecology and Obstetrics (FIGO) initiative on gestational diabetes mellitus: a pragmatic guide for diagnosis, management, and care. Int J Gynaecol Obstet. 2015;131:S3. age appropriate intake of vegetables and fruits, whole grain cereal, and starch, while discouraging excess intake of fat and $\mathrm{Na}^{+}$-rich foods.

This combined with the signal system, plate model, food journal, and food exchange tables helps empower patients to understand and adapt healthy eating behaviour. Almost all guidelines recommend health education sessions and using the services of a dietician to give MNT [11]. Availability of trained dieticians maybe a concern in many developing and low-resource countries, but this shortfall can be overcome by training other health care workers to give focussed guidance on healthy eating using some of the principles and tools described above.

\section{Conflict of Interest Statement}

The writing of this article was supported by Nestlé Nutrition Institute, and the authors declare no other conflicts of interest.

10 Artal R, Catanzaro RB, Gavard JA, Mostello DJ, Friganza JC. A lifestyle intervention of weight-gain restriction: diet and exercise in obese women with gestational diabetes mellitus. Appl Physiol Nutr Metab. 2007;32(3):596-601.

11 Tsirou E, Grammatikopoulou MG, Theodoridis X, Gkiouras K, Petalidou A, Taousani E, et al. Guidelines for medical nutrition therapy in gestational diabetes mellitus: systematic review and critical appraisal. J Acad Nutr Diet. 2019;119(8):1320-39.

12 Prenatal Nutrition Guidelines for Health Professionals. Gestational weight gain. Health Canada. 2010.

13 Moreno-Castilla C, Mauricio D, Hernandez M. Role of medical nutrition therapy in the management of gestational diabetes mellitus. Curr Diab Rep. 2016;16(4):22

14 Muktabhant B, Lawrie TA, Lumbiganon P, Laopaiboon M. Diet or exercise, or both, for preventing excessive weight gain in pregnancy. Cochrane Database Syst Rev. 2015; (6): CD007145.

15 Vestgaard M, Christensen AS, Viggers L, Lauszus FF. Birth weight and its relation with medical nutrition therapy in gestational diabetes. Arch Gynecol Obstet. 2017 Jul;296(1):35-41.

16 Shepherd E, Gomersall JC, Tieu J, Han S, Crowther CA, Middleton P. Combined diet and exercise interventions for preventing gestational diabetes mellitus. Cochrane Database Syst Rev. 2017;11: CD010443.

17 ACOG Practice Bulletin No. 180. Practice bulletin No. 180 summary: gestational diabetes mellitus. Obstet Gynecol. 2017;130(1):244-6.

18 Blumer I, Hadar E, Hadden DR, Jovanovič L, Mestman JH, Murad $\mathrm{MH}$, et al. Diabetes and pregnancy: an Endocrine Society clinical practice guideline. J Clin Endocrinol Metab. 2013;98(11):4227-49. 
19 Romon M, Nuttens MC, Vambergue A, Vérier-Mine O, Biausque S, Lemaire $C$, et al. Higher carbohydrate intake is associated with decreased incidence of newborn macrosomia in women with gestational diabetes. J Am Diet Assoc. 2001;101(8):897-902.

20 Looman M, Schoenaker DAJM, Soedamah-Muthu SS, Geelen A, Feskens EJM, Mishra GD. Pre-pregnancy dietary carbohydrate quantity and quality, and risk of developing gestational diabetes: the Australian longitudinal study on women's health. $\mathrm{Br} \mathrm{J}$ Nutr. 2018;120(4):435-44.

21 Barbour LA, Hernandez TL. Maternal lipids and fetal overgrowth: making fat from fat. Clin Ther. 2018;40(10):1638-47.

22 Olmos PR, Rigotti A, Busso D, Berkowitz L, Santos JL, Borzone GR, et al. Maternal hypertriglyceridemia: a link between maternal overweight-obesity and macrosomia in gestational diabetes. Obesity. 2014;22(10):2156-63.

23 Hernandez TL, Van Pelt RE, Anderson MA, Reece MS, Reynolds RM, de la Houssaye BA, et al. Women with gestational diabetes mellitus randomized to a higher-complex carbohydrate/low-fat diet manifest lower adipose tissue insulin resistance, inflammation, glucose, and free fatty acids: a pilot study. Diabetes Care. 2016;39(1): $39-42$.

24 Hernandez TL, Mande A, Barbour LA. Nutrition therapy within and beyond gestational diabetes. Diabetes Res Clin Pract. 2018;145: $39-50$.

25 Bao W, Bowers K, Tobias DK, Olsen SF, Chavarro J, Vaag A, et al. Prepregnancy low-carbohydrate dietary pattern and risk of gestational diabetes mellitus: a prospective cohort study. Am J Clin Nutr. 2014;99(6):1378-84.

26 Augustin LS, Kendall CW, Jenkins DJ, Willett WC, Astrup A, Barclay AW, et al. Glycemic index, glycemic load and glycemic response: an international scientific consensus summit from the International Carbohydrate Quality Consortium (ICQC). Nutr Metab Cardiovasc Dis. 2015;25(9):795-815

27 Brand-Miller J, Hayne S, Petocz P, Colagiuri S. Low-glycemic index diets in the management of diabetes: a meta-analysis of randomized controlled trials. Diabetes Care. 2003;26(8):2261-7.

28 Nolan CJ. Improved glucose tolerance in gestational diabetic women on a low fat, high unrefined carbohydrate diet. Aust N Z J Obstet Gynaecol. 1984;24(3):174-7.

29 Cypryk K, Kamińska P, Kosiński M, Pertyńska-Marczewska M, Lewiński A. A comparison of the effectiveness, tolerability and safety of high and low carbohydrate diets in women with gestational diabetes. Endokrynol Pol. 2007;58(4):314-9.

30 Moses RG, Barker M, Winter M, Petocz P, Brand-Miller JC. Can a low-glycemic index diet reduce the need for insulin in gestational diabetes mellitus? A randomized trial. Diabetes Care. 2009; 32(6): $996-1000$.

31 Asemi Z, Tabassi Z, Samimi M, Fahiminejad T, Esmaillzadeh A. Favourable effects of the dietary approaches to stop hypertension diet on glucose tolerance and lipid profiles in gestational diabetes: a randomised clinical trial. Br J Nutr. 2013;109(11):2024-30.

32 Lauszus FF, Rasmussen OW, Henriksen JE, Klebe JG, Jensen L, Lauszus KS, et al. Effect of a high monounsaturated fatty acid diet on blood pressure and glucose metabolism in women with gestational diabetes mellitus. Eur J Clin Nutr. 2001;55(6):436-43.
33 Wei J, Heng W, Gao J. Effects of low glycemic index diets on gestational diabetes mellitus: a meta-analysis of randomized controlled clinical trials. Medicine. 2016;95(22):e3792.

34 Filardi T, Panimolle F, Crescioli C, Lenzi A, Morano S. Gestational diabetes mellitus: the impact of carbohydrate quality in diet. Nutrients. 2019;11(7):1549.

35 Zhang R, Han S, Chen GC, Li ZN, Silva-Zolezzi I, Parés GV, et al. Effects of low-glycemic-index diets in pregnancy on maternal and newborn outcomes in pregnant women: a meta-analysis of randomized controlled trials. Eur J Nutr. 2018;57(1):167-77.

36 Viana LV, Gross JL, Azevedo MJ. Dietary intervention in patients with gestational diabetes mellitus: a systematic review and metaanalysis of randomized clinical trials on maternal and new born outcomes. Diabetes Care. 2014;37:3345-55.

37 Han S, Middleton P, Shepherd E, Van Ryswyk E, Crowther CA. Different types of dietary advice for women with gestational diabetes mellitus. Cochrane Database Syst Rev. 2017;2(2):CD009275.

38 Markovic TP, Muirhead R, Overs S, Ross GP, Louie JC, Kizirian N, et al. Randomized controlled trial investigating the effects of a low-glycemic index diet on pregnancy outcomes in women at high risk of gestational diabetes mellitus: the Gl baby 3 study. Diabetes Care. 2016;39(1):31-8.

39 Schaefer-Graf UM, Graf K, Kulbacka I, Kjos SL, Dudenhausen J, Vetter $\mathrm{K}$, et al. Maternal lipids as strong determinants of fetal environment and growth in pregnancies with gestational diabetes mellitus. Diabetes Care. 2008;31(9):1858-63.

40 Eslamian L, Akbari S, Marsoosi V, Jamal A. Effect of different maternal metabolic characteristics on fetal growth in women with gestational diabetes mellitus. Iran J Reprod Med. 2013;11(4):32534

41 Hernandez TL, van Pelt RE, Anderson MA, Daniels LJ, West NA, Donahoo WT, et al. A higher-complex carbohydrate diet in gestational diabetes mellitus achieves glucose targets and lowers postprandial lipids: a randomized crossover study. Diabetes Care. 2014;37(5):1254-62.

42 Asemi Z, Samimi M, Tabassi Z, Esmaillzadeh A. The effect of DASH diet on pregnancy outcomes in gestational diabetes: a randomized controlled clinical trial. Eur J Clin Nutr. 2014;68(4):490-5.

43 Assaf-Balut C, García de la Torre N, Durán A, Fuentes M, Bordiú E, del Valle $L$, et al. A Mediterranean diet with additional extra virgin olive oil and pistachios reduces the incidence of gestational diabetes mellitus (GDM): a randomized controlled trial: the St. Carlos GDM prevention study. PLoS One. 2017;12(10):e0185873.

44 Olmedo-Requena R, Gómez-Fernández J, Amezcua-Prieto C, Mozas-Moreno J, Khan KS, Jiménez-Moleón JJ. Pre-pregnancy adherence to the Mediterranean diet and gestational diabetes mellitus: a case-control study. Nutrients. 2019;11(5):1003.

45 de la Torre NG, Assaf-Balut C, Jiménez Varas I. Effectiveness of following Mediterranean diet recommendations in the real world in the incidence of gestational diabetes mellitus (GDM) and adverse maternal-foetal outcomes: a prospective, universal, interventional study with a single group. The St Carlos study. Nutrients. 2019;11(6):1210.

46 Committee on Practice Bulletins - Obstetrics. ACOG practice bulletin no. 190: gestational diabetes mellitus. Obstet Gynecol. 2018;131:e49-64. 
47 Agha-Jaffar R, Oliver N, Johnston D, Robinson S. Gestational diabetes mellitus: does an effective prevention strategy exist? Nat Rev Endocrinol. 2016;12(9):533-46.

48 Tieu J, Shepherd E, Middleton P, Crowther CA. Dietary advice interventions in pregnancy for preventing gestational diabetes mellitus. Cochrane Database Syst Rev. 2017;1:CD006674.

49 Donazar-Ezcurra M, López-Del Burgo C, Bes-Rastrollo M. Primary prevention of gestational diabetes mellitus through nutritional factors: a systematic review. BMC Pregnancy Childbirth. 2017; 17(1):30.

50 Luoto R, Laitinen K, Nermes M, Isolauri E. Impact of maternal probiotic-supplemented dietary counselling on pregnancy outcome and prenatal and postnatal growth: a double-blind, placebo-controlled study. Br J Nutr. 2010;103(12):1792-9.

51 Zhang H, Lv Y, Li Z, Sun L, Guo W. The efficacy of myo-inositol supplementation to prevent gestational diabetes onset: a metaanalysis of randomized controlled trials. J Matern Fetal Neonatal Med. 2018:1-7.

52 Mahajan A, Donovan LE, Vallee R, Yamamoto JM. Evidencedbased nutrition for gestational diabetes mellitus. Curr Diab Rep. 2019;19(10):94.
53 Anjana RM, Vijayalakshmi P, Bhavadharini B, Gayathri R, Lakshmipriya N, Uthra S. Association of whole grains, dairy and dietary fibre with neonatal outcomes in women with gestational diabetes mellitus: the WINGS project (WINGS-12). J Diabetol. 2019;10: 127-33.

54 Hrolfsdottir L, Gunnarsdottir I, Birgisdottir BE, Hreidarsdottir IT, Smarason AK, Hardardottir $\mathrm{H}$, et al. Can a simple dietary screening in early pregnancy identify dietary habits associated with gestational diabetes? Nutrients. 2019;11(8):1868

55 Kapur K, Kapur A, Ramachandran S, Mohan V, Aravind SR, Badgandi $M$, et al. Barriers to changing dietary behavior. J Assoc Physicians India. 2008;56:27-32.

56 Kapur K, Kapur A. The signal system: an empowering tool for healthy food choices. IDF Diabetes Voice. 2005;50(2).

57 Booth K, Youde S, Bennett T; Sydney Diabetes, Northern Sydney Central Coast Health (NSW). The traffic light guide to food: type 2 diabetes and gestational diabetes. 7th ed. St. Leonards, NSW: Diabetes Education Centre, RNSH; 2010.

58 Raidl M, Spain K, Lanting R, Lockard M, Johnson S, Spencer M, et al. The healthy diabetes plate. Prev Chronic Dis. 2007;4(1):A12

59 Shi M, Liu ZL, Steinmann P, Chen J, Chen C, Ma XT, et al. Medical nutrition therapy for pregnant women with gestational diabetes mellitus: a retrospective cohort study. Taiwan J Obstet Gynecol 2016;55(5):666-71. 